pared for filing under the proper places. Such a work carefully executed would eliminate at once almost all the changes in nomenclature due to priority only, the names, that seem to irritate most grievously the men who are not actually engaged in revisional work.

The reviser usually has only one aim, or should have only one aim in mind, and that is to achieve stability by applying the rules of the international code consistently, no matter how much he may dislike to do so. No nomenclatorial stability can be achieved if each of us follows an independent method. A catalogue of the kind above referred to would make a quick revision possible, the main points of which would stand for a long time to come, and the minor shift could easily be kept current by the small force that should prepare the cards for the new things published year by year. I wish to heartily recommend this undertaking to the National Research Council. I am sure that the whole zoological fraternity, yes, not only zoological but botanical fraternity, would be grateful for such a work.

It is to be hoped that Professor Morse will continue this work and will find time to give us the results of his efforts.

\section{Paul Bartsch}

\section{VENOMOUS SPIDERS}

MY attention having recently been called to the death of a man, apparently from the bite of a spider (which case will be described below), I have brought together some of the literature upon this much debated question, and I shall quote from several authorities upon the subject.

Comstock, in "The Spider Book," makes the following statements in discussing the venomous character of spider bites:

During my study of spiders I have collected thousands of specimens and have taken very many in my hand but have never been bitten by one (p. 213).

Several of the more prominent arachnologists, including Mr. Blackwall, of England, and Baron Walckenaer and M. Duges, of France, have made experiments to determine the effect on man of the bite of spiders. Each of these experimenters caused himself to be bitten by spiders; and all agree that the effects of the lbites did not differ materially from those of pricks made the same time with a needle (p. 214).

I have given considerable attention to this question with the result that I firmly believe that in the North at least there is no spider that is to be feared by man.

Although we have in the North no spider that is to be feared, it is quite possible that in the South it is different. I confess that I should not like to be bitten by one of the larger tarantulas of that region, although I know of no well-authenticated case of a person being bitten by one.

The spiders of the genus Latrodectus, of which we have a common representative in the South, are feared wherever they oceur, and it is possible that they are more venomous than other spiders. . . .

This genus, as has been woll stated by F. P. Cambridge, comprises those very interesting spiders which, under various local names, have been notorious in all ages and in all regions of the world where they occur on account of the reputed deadly nature of their bite. It may be added that this belief is not shared by students of spiders . . . (p. 357).

This species ( $L$. mactens) is very common and widely distributed in the South. It is found under stones and pieces of wood on the ground, about stumps, in holes in the ground, and about outbuildings ... (p. 358).

Although it is essentially a southern species, it occurs in Indiana, Ohio, Pennsylvania, New Hampshire and doubtless other of the northern states. ... It also occurs in California (p. 358).

An apparent inconsistency is seen in the above quotations. He states in one place "that in the north at least there is no spider that is to be feared by man." A little later he says:

Although it (Latrodectes) is essentially a southern species, it occurs in Indiana, Ohio, Pennsylvania, New Hampshire, and doubtless other of the nonthern states. ...

Since he reports Latrodectes from Pennsylvania and New Hampshire it is obviously not an entirely southern species.

Long before the publication, in 1912, of "The Spider Book," in Vol. 1, 1889, of Insect Life, the editors, Riley and Howard, discussed in two articles, the question of spider bites. 
In the first of these, "A Contribution to the Literature of Fatal Spider Bites," various cases are reported, one of which was fatal in fourteen hours. They call attention to the fact that the genus Latrodectes, under various local names, in widely separated parts of the world, has the reputation of being poisonous; it is even classed, in this respect, with the rattlesnakes, by some Indian tribes. This very widespread reputation would seem to be fair evidence in favor of the view that this spider has marked poisonous characters.

After discussing the above-mentioned cases, the authors make the following statement:

... it seems to us, after analyzing the evidence, that it must at least be admitted that certain spiders of the genus Latrodectes have the power to inflict poisonous bites, which may (probably exceptionally and depending upon exceptional condition's) bring about the death of a human being (p. 211).

In a later communication, "The Spider Bite Question Again," in the same volume of Insect Life, the editors quote a letter from Dr. E. R. Corson, of Savannah, Georgia, in which four cases are described in detail of supposed bites from spiders, though in no case was the animal actually captured or seen. Two of these cases were adult colored men; one was a very strong, healthy white man; the fourth was a two-year-old boy. None of these victims died, though some of them were most seriously affected, the symptoms being practically the same in each case as those to be described below for the two cases under discussion. As in these two, and practically every case recorded, the victims were bitten on the penis while using an outdoor closet.

U. L. Kellogg' in an article entitled "Spider Poison" discussed the subject of bites from Latrodectes in a most interesting manner. He quotes the same statement from Comstock, given above, in regard to the skepticism of students of spiders as to the serious nature of their bites; and he says

To this I may in turn add that at least one student of spiders, though incomparably less experi-

1 Journal of Parasitology, Vol. 1, 1915, pp. 107112. enced than Comstock, does share the belief in the unusually poisonous nature of Latrodectes mactens.

The chief case cited by Kellogg is one that he quotes from one of his former students, Dr. Coleman. It will be noted that in this case the spider was captured and identified as L. mactans. The case is as follows, in Dr. Coleman's words:

Patient B came to my office one morning at 8.15 0 'clock, showing signs of an acute poisoning of some sort.

The glans of the penis had been bitten by a spider while the patient was sitting in an outcloset. The only thing felt was a sharp sting. (The spider was eaptured, so there is no doubt as to the species; it was a female of Latrodectes mactens.) In about ten minutes there appeared dizziness and weakness of the legs, followed by cramps in the abdominal muscles.

The patient left the field where working and started to walk to town, a distance of a little over a mile. The pains grew worse and the penis started to swell and turn red. When the office was reached, the pains, of a cramp-like character, in the abdomen, were intense, also around the heart and thighs. Physical examination showed the heart to be running at a rate of 40 per minute, of small volume but regular. The respiration was labored. The pupils were dilated and the face very red and congested. The penis was swollen to a great size, fully three inches in diameter at the glans, and the color was a mottled purple. The contractions were clonic in character, giving the greatest pain in the chest and abdomen. There were no pains below the knees or elbows.

The treatment consisted of hypodermic injections strychnin $1 / 40$, followed in ten minutes by nitroglycerine 1/100. Local applications to the site of bite of the crystals of potassium permanganate. The heart went as low as 27 beats to the minute. After three hours' work, using repeated injections of strychnin, the heart-rate was increased to $\mathbf{4 5}$. The pains were not quite so severe and the patient was taken home. The administration of strychnin was stopped and the use of brandy hypodermically injected was substituted, a dose of $10 \mathrm{~mm}$. being given every hour. Heat was applied to the feet and back. At 5 P.M., or about nine and one half hours after the first symptoms, the heart-rate had been raised to 55 and then as the pains were still severe, a morphin with $1 / 150$ atropin was given. The pains eased up and the patient dropped 
The next morning a fine rash appeared all over the body, accompanied by some itching. The penis had returned to nearly normal size. The heartrate was 60 , the respiration was 18 and deep, temperature 100. The rash disappeared in four days. The patient was troubled with insomnia for several days, and a stubborn constiplation that took a very active purge to affect. . . .

Dr. Coleman also tried various experiments with spider poison, both upon himself and upon lower mammals; two of these are as follows:

Several experiments were tried on rabbits and cats with very interesting results.

1. The dissected glandis of one female Latrodectes containing the virus. The virus was macerated in 10 drops of distilled water. The same was injected subcutaneoúsly into the abdomen of a cat about 8 months old. In about five minutes a series of convulsions set in of a clonic type, quickly followed by tonic spasm and in ten minutes the animal was dead.

3. A quantity of the eggs of Latrodectes was macerated in 20 gtts. of water and diluted up to 10 e.e. The injection of this solution produced the same typical symptoms and death to a cat 8 months old in abrout three minutes. A rabbit was killed in about. two and one half minutes. ...

It will be noted that the spider poison or "arachnolysin" is not confined to the poison glands but is found in the body fluids and tissues as well. Kellogg says:

A diadem spider of $1.4 \mathrm{gr}$. contains sufficient poison to destroy completely all the corpuscles in 2.5 liters of rabbit blood. This puts arachnolysin in the class of strongest kinds of blood poisons.

He says also:

Probably with Latrodectes, as with other animal poisons, the physiological idiosyncrasies of the particular man ibitten play an important part in determining the degree of seriousness of the trouble produced.

The most recent case brought to my attention, by the sister of the victim, occurred in Oklahoma in the summer of 1920 . The victim was a strong healthy man of thirty-eight; he died about thirty-two hours after being bitten, in spite of the efforts of three physicians. All three of the doctors were written to for details of the case, but only one Dr. E. W. Reynolds, responded. His description of the case, so far as he knew it, follows.

I was called on the case shortly after Mr. L. was bitten. He had gone to the toilet and while sitting on the stool felt something like a pin stick him but did not look until he was bitten again and then discovered a little black spider which he killed. . . .

He was bitten on the end of the penis and I could not see any marks or swellings which probably meant that he received the poison directly into a small blood vessel.

When I saw him he was in great agony. The pain traveled up the penis through the cords to one group of muscles and another, shifting about all the time. The usual amount of narcotic had no effect at all. I was with him about one hour but did not get to see him again. He could not lie still, and when I left he was some easier and not depressed. When I left I expected to have to go back later and give him another hypo but wanted to wait awhile to see what effect it already had.

For some reason later other doctors were called but I understand were unable to help him in any way.

It will be noticed that in this case also the victim was bitten on the penis while using a toilet, and that a "little black spider" was killed.

It would seem worth while for the public in general, especially in the rural districts, to become familiar with this rather formidable little animal, whose colors and markings make it easy to recognize.

Since writing the above I have received from Dr. V. L. Casto, the father of one of my students, the following history:

On the 30th day of last October I was ealled to see Andy Coon, age 48, farmer and American, who about two hours previous to my visit was bitten by a small spider, black in color: he had been husking corn and had noticed several small black spiders in the fodder and one of them seemed to get tangled in his underwear and bit him four times, about five inches above the left ankle.

I found him in the following condition: suffering excruciating pain radiating from the place of the bite to the top of his head; the leg swollen, a severe pain around the heart, pulse 140 per minute, and in about 30 minutes the pain passed to the opposite side and the left leg and thigh began to swell and he began to swell over the region of the 
kidneys; at this stage he began to vomit and went into collapse and broke out into a cold and clammy sweat, remaining this way for two hours.

$\mathrm{He}$ continued to swell for 12 hours, when it stopped, and it was 48 hours before the swelling began to leave and six weeks after the bite the patient still complains of soreness in his legs and some pain around his heart, yet he is able to resume his work on the farm.

Also, since writing the above, an article has appeared in the Journal of the American Medical Association for January 8, 1921, page 99 , by Dr. D. J. Lewis of San Juan, Coahuila, Mexico, entitled "Black Spider Poisoning; a Report of Four Cases.". In this article he briefly describes the cases of three men, aged respectively 31,32 , and 33 years, and of one woman, aged 28 , all of whom were bitten while asleep in bed at night. Dr. Lewis states that gauze wet with saturated solution of magnesium sulphate kept on the bitten area "relieves the pain, reduces the swelling and prevents the progress of the disease." $\mathrm{He}$ also gave iodine, calomel and magnesium sulphate internally, but he does not state in what doses. The patients were able to resume work in from five to ten days.

WEST VIRGINIA UNIVERSITY

AlberT M. REESF

\section{SPECIAL ARTICLES}

PREVALENCE AND DISTRIBUTION OF FUNGI INTERNAL OF SEED CORN

THE importance of root, stalk and ear rot fungi in decreasing yields of field corn has received considerable attention in recent years on the part of investigators. Results of investigations so far reported indicate more or less agreement in the various disease symptoms manifested. However, some difference of opinion exists concerning the importance of the causal organisms. The specific determination of the fungi has not been fully emphasized nor the method by which they are carried in the seed.

The following account presents in part the results of our investigations in determining the species of fungi associated with seed corn.
Our studies were initiated to ascertain the losses and prevalence of infection in Delaware and the importance of the seed in carrying infection. While our observations and studies have been confined principally to the corn crop in this state we feel that careful investigations will reveal the presence and importance of the same pathogenes in other states but no doubt in varying degrees of prevalence.

Disinfection experiments followed by cultures soon indicated to us that certain parasitic fungi were carrying internal of the kernel and that a brief surface sterilizing with a strong disinfectant, followed by proper culture methods proved an efficient means of determining the amount of such internal infection.

We have found that an efficient test for determining the presence of fungi internal of seed corn and one which at the same time readily permits of the identification of the fungi, is carried out by disinfecting and planting the kernels or crushed kernels in sterile culture medium in Petri dishes. Fifteen or more kernels are disinfected in a test tube $150 \times 20 \mathrm{~mm}$. for one minute in a solution of 50 per cent. alcohol containing 1 gram of bichloride of mercury in each liter. Following this treatment the kernels are washed in the same tube with two successive washings with 20 c.c., each of sterile water and immediately ten kernels are removed aseptically with sterile forceps and placed with germ side down on 20 c.c., of nutrient glucose agar in a sterile culture dish. Further, five of the remaining kernels are each placed in a sterile culture dish and with a sterile scalpel the point of the kernel which is the portion that contains most of the internal infection is cut off one sixth to one fifth inch from the end; then with a strong sterile forceps each point is placed in the mouth of a heavy-walled tube (it requires a strong tube and strong forceps, as crushing is not easy) $150 \times 20 \mathrm{~mm}$. containing 10 c.c., of sterile nutrient glucose agar medium at $43^{\circ} \mathrm{C}$; ; the point is thoroughly crushed and shaken down into the medium, then well 